\title{
Das 500. Reformationsjubiläum in rumänisch-orthodoxer Wahrnehmung. Barometer für den aktuellen Stand der Ökumenischen Bewegung?
}

\author{
Hans Bruno FröHLICH ${ }^{*}$
}

In my article I would like to document assessments from the 500th anniversary of the Reformation in the perception of the Romanian Orthodoxy. From these evaluations, conclusions about the current position of Orthodoxy to Protestantism can be drawn. The focus is on a total of seven contributions. Two are from the online news portal of the Bucharest Patriarchate www.basilica.ro by Gheorghe Anghel and Aurelian Iftimiu. Three articles were published in the "Revista Teologică" from Theological Faculty in Sibiu by Daniel Buda \& Aurel Pavel, Mihai Iordache and Maria Curtean. Finally, Im referencing to two contributions published by the Bucharest theologian Cosmin Pricop in German-language publications.

Keywords: 500 th Anniversary of the Reformation, Orthodox Perception of the Reformation, Orthodoxy and Protestantism, Ecumenical Movement

I.

Dieser Beitrag ist in gekürzter Form auf einer Tagung der „Lucian Blaga“Universität vorgetragen worden, die vom 31. Oktober bis 3. November 2019 in der Evangelischen Akademie in Hermannstadt stattgefunden hat und die Überschrift „Grenzen überschreiten: 500 Jahre Reformation in Hermannstadt/Siebenbürgen - 70 Jahre Protestantisch-Theologisches Institut" trug. Die Schnittmenge zwischen der Thematik jener Tagung und der aktuellen Ausgabe von RES zum Thema »Ökumenische Bewegung: Quo Vadis? Überlegungen zur Ökumene in Zentral- und Osteuropa« besteht darin, dass sowohl die Reformation als auch die Ökumenische Bewegung etwas mit Grenzüberschreitungen zu tun haben, ja mehr noch: Beide definieren sich geradezu durch Grenzüberschreitungen. Eine dieser Grenzüberschreitung ist auch die Reaktion der Orthodoxie allgemein und der rumänischen Orthodoxie im Speziellen auf das Reformationsjubiläum.

\footnotetext{
* Dr. Hans Bruno Fröhlich, Stadtpfarrer und Dechant in Schäßburg, str. Cositorarilor 13, RO-545400 Sighişoara, bruno@schaessburg.ro.
} 
Das 500. Reformationsjubiläum in rumänisch-orthodoxer Wahrnehmung

Das heutige Rumänien ist in mancherlei Hinsicht ein „Grenzland Europas" "1; so auch hinsichtlich seiner religiösen bzw. konfessionellen Geschichte und Struktur. Einerseits gibt es gerade in den multiethnischen und -konfessionellen bzw. -religiösen Regionen (Siebenbürgen, Banat, Bukowina oder Dobrudscha) eine jahrhundertealte Tradition der Koexistenz von Religionen und Konfessionen. Andererseits aber muss diese Koexistenz natürlich in den jeweiligen historischen Gegebenheiten gesehen und bewertet werden. Seit gut 100 Jahren ist - so Hans Küng - die Christenheit ins „ökumenischen Paradigma“2 eingetreten. Die Entwicklung in dieser Zeitspanne ist bemerkenswert. Vor gut 100 Jahren noch - im Jahr 1917 wurde das 400. Reformationsjubiläum gefeiert - hätte niemand eine solche Feier in Zusammenhang mit der Orthodoxie gebracht. Fünfzig Jahre später war das bereits anders. In der Dezemberausgabe 2017 von RES, in der die Tagung des Institutes für Ökumenische Forschung vom Mai 2017 zum Thema "Reformation und Orthodoxie“ dokumentiert wird, liefert Dragoș Boicu in seinem Aufsatz: „An Almost Forgotten Jubilee - The 450th Anniversary of the Reformation through the Pages of Romanian Orthodox Press" einen bemerkenswerten Einblick in die Wahrnehmung des 450. Reformationsjubiläums aus dem Jahr 1967. ${ }^{3}$

In meinem Beitrag möchte ich versuchen, ein paar Streiflichter über das 500. Reformationsjubiläum in der Wahrnehmung der rumänischen Orthodoxie zu dokumentieren. Gerade weil die sozialen, politischen und historischen Umstände zwischen den Jahren 1967 und 2017 sich grundlegend gewandelt haben, finde ich es herausfordernd, einen Blick auf die jetzige Wahrnehmung der Reformation durch die Orthodoxie zu werfen. Aus diesen orthodoxen Bewertungen - die, wie wir sehen werden, unterschiedlich ausfallen - können Rückschlüsse zur heutigen Position der Orthodoxie dem Protestantismus allgemein und dem Luthertum im Speziellen gegenüber gezogen werden. Mit diesem Artikel möchte ich aber auch einen bescheidenen Beitrag dazu leisten, dass die Wahrnehmung der beiden letzten Jubiläen in Relation zueinander gesetzt werden können, damit - anhand von Ähnlichkeiten und Unterschieden - auch Rückschlüsse auf die Entwicklung der Bewertung der Reformation durch die Orthodoxie gezogen werden können.

${ }^{1}$ Lucian Boia, România. Țară de frontieră a Europei, București, Humanitas 2007.

2 Hans Küng, Das Christentum - Die religiöse Situation der Zeit, München - Zürich, Piper 1999 .

3 Dragoş Boicu, „An Almost Forgotten Jubilee - The 450th Anniversary of the Reformation through the Pages of Romanian Orthodox Press", in: Review of Ecumenical Studies 9 (3/2017), S. 394-409. 
Mein Ziel ist nicht, die Wahrnehmung des 500. Reformationsjubiläums in der rumänischen Orthodoxie exhaustiv zu beschreiben. Andererseits ist auch die Zielsetzung der von mir durchgesehenen Medien in erster Linie jene, innerorthodoxe Themen zu behandeln, so dass eine flächendeckende Thematisierung von Reformation und Protestantismus sowieso nicht erwartet werden kann. Ich werde mich zunächst auf zwei Beiträge des Onlineportals www.basilica.ro des Bukarester Patriarchates fokussieren, dann drei Beiträge aus der in Hermannstadt herausgegebenen Zeitschrift „Revista Teologică“ analysieren und schließlich zwei Artikel des jungen Theologen Cosmin Pricop, die in deutschen Fachzeitschriften publiziert wurden, in meine Betrachtungen einfließen lassen.

\section{II.}

Die Webseite www.basilica.ro ist eine wichtige Stimme des Rumänisch-Orthodoxen Patriarchates, wobei es sich hier nicht um eine theologische Fachzeitschrift, sondern um ein Nachrichtenportal handelt. Am 5. November 2017, also eine knappe Woche nach dem 500. Reformationsjubiläum, erschien auf dieser Seite ein bemerkenswerter Artikel, herausgegeben vom Pressebüro des Rumänischen Orthodoxen Patriarchates und verfasst von Gheorghe Anghel: „Diferiți în credință, dar pașnici în conviețuire: Ortodocși și luterani în Transilvania“4 („Verschieden im Glauben, aber friedlich im Zusammenleben: Orthodoxe und Lutheraner in Siebenbürgen"). Einerseits wird auf die geschichtliche und kirchenpolitische Relevanz dieses Ereignisses Bezug genommen. Andererseits aber finden wir hier eine telegrammstilartige, präzise theologische Einschätzung der Reformation in orthodoxer Sicht vor.

Der Autor verweist ganz am Anfang seines Beitrags auf den Appell zur gegenseitigen Vergebung zwischen Protestanten und Katholiken, so wie dies aus der Gemeinsamen Stellungnahme des Lutherischen Weltbundes und des Päpstlichen Rates zur Förderung der Einheit der Christen zum Abschluss des gemeinsamen Reformationsgedenkjahres am 31. Oktober 2017 hervorgeht: „Ebenso haben wir auch um Vergebung gebeten für unser Versagen und dafür, wie Christen und Christinnen in den fünfhundert Jahren seit Beginn der Reformation bis heute den Leib des Herrn verletzt und einander gekränkt haben. "5 Anghel ist der Meinung, dass dieses Ereignis ein Anlass dafür ist,

\footnotetext{
${ }^{4}$ https://basilica.ro/diferiti-in-credinta-dar-pasnici-in-convietuire-ortodocsi-si-luterani-in-transilvania/, abgerufen am 21.09.2019.

5 https://de.lutheranworld.org/sites/default/files/documents/171031gemeinsame_stellungsnahme_de.pdf, abgerufen am 28.09.2019.
} 
Das 500. Reformationsjubiläum in rumänisch-orthodoxer Wahrnehmung

die innerchristlichen orthodox-lutherischen Beziehungen allgemein und die historischen Beziehungen zwischen der Rumänischen Orthodoxen Kirche und der Evangelisch-Lutherischen Kirche in Siebenbürgen hervorzuheben. ${ }^{6}$ Dem Leser werden zwei wichtige historische Momente in Erinnerung gerufen, welche die geschwisterlichen und freundschaftlichen Beziehungen zwischen den beiden Kirchen bzw. zwischen Persönlichkeiten dieser beiden Kirchen in ganz besonderer Weise hervorheben: die Zusammenarbeit der siebenbürgischen Reformatoren mit rumänisch-orthodoxen Buchdruckern wie Philip Moldner (Filip Moldoveanu) oder dem Diakon Coresi und jene zwischen den beiden großen Persönlichkeiten den 19. Jahrhunderts, nämlich dem im Jahr 2011 heiliggesprochenen Metropoliten Andrei Șaguna und dem Sachsenbischof Georg Daniel Teutsch. Eine Folge dieser jahrhundertealten fruchtbaren und gedeihlichen Zusammenarbeit ist auch der akademische interkonfessionelle bilaterale Dialog, den die Rumänische Orthodoxe Kirche mit der Evangelischen Kirche in Deutschland bis auf den heutigen Tag führt. Dieser Dialog begann 1979 in Goslar und feierte vergangenes Jahr sein 40-jähriges Bestehen: vom 29. Juli - 1. August 2019 wurde er zum 15. Mal unter dem Thema „Die missionarischen Herausforderungen unserer Kirchen in der säkularisierten Gesellschaft "7 im Kloster Caraiman (Bușteni) abgehalten.

Aus Anghels Einschätzungen kann man ableiten, dass die Beziehungen zwischen Orthodoxen und Lutheranern in Rumänien bzw. Siebenbürgen auf eine jahrhundertealte Tradition blicken, die es - im Gegensatz zur Beziehung mit der Katholischen Kirche - gar nicht erfordern, um Vergebung bitten zu müssen, da es Krieg und Gewalt aus religiösen Gründen hier nicht gegeben hat. In dem kurzen theologischen Teil seines Artikels arbeitet er in präziser Weise den Konflikt zwischen Martin Luther und der damaligen katholischen Kirche heraus, wobei eine gewisse Sympathie des Autors für Luthers theologische Argumente und eine gewisse Antipathie für die (weniger theologische) Reaktion der Kurie darauf nicht zu übersehen sind. Das Schisma von 1054 erwähnend, entwickelt Anghel seine Argumentation folgendermaßen: Luther stellt der Macht des Papstes und der kirchlichen Tradition die Autorität der Heiligen Schrift entgegen (sola scriptura), die

\footnotetext{
${ }^{6}$ „Acest eveniment constituie și un prilej de evidențiere a relațiilor intercreștine ortodoxe-luterane, în general, și a relațiilor istorice dintre Biserica Ortodoxă Română și Biserica Evanghelică Luterană din Transilvania, în particular.“ Siehe: https://basilica.ro/diferiti-incredinta-dar-pasnici-in-convietuire-ortodocsi-si-luterani-in-transilvania/, abgerufen am 21.09.2019.

7 https://www.ekd.de/40-jahre-dialog-rumaenisch-orthodoxe-kirche-48538.htm, abgerufen am 21.09.2019.
} 
er in die Volkssprache übersetzt; Luther ersetzt die Lehre von den guten Werken mit der Genügsamkeit des Glaubens (sola fide) und der Gnade (sola gratia); Luther ersetzt das durch Weihe übertragene Priestertum mit dem allgemeinen Priestertum aller Getauften; und schließlich ersetzt Luther die scholastische Theologie durch eine Schrifttheologie, wobei er den Heiligenund Bilderkult verwirft. Die Auseinandersetzungen innerhalb des westlichen Christentums, welche nicht selten gewalttätig ausgetragen wurden und sogar in Kriegen ausgeartet sind, fanden erst im 20. Jahrhundert ein Ende, als die Polemik durch den ökumenischen Dialog ersetzt wurde. Dass die beiden konkurrierenden Kirchen des Westens immer wieder versucht haben, die Orthodoxie auf ihre Seite zu ziehen, ist im aktuellen ökumenischen Kontext nur noch eine Randnotiz, die aber in der geschichtlichen Entwicklung eine wichtige Rolle gespielt hat.

Der Artikel schließt mit einer Bemerkung, die auch als Zusammenfassung des letzten bilateralen Dialoges zwischen der Rumänischen Orthodoxen Kirche und der Evangelischen Kirche gelten könnte:

In einem zunehmend säkularisierten, angespannten und fragmentierten Europa ist ein gemeinsames Bekenntnis der ewigen Werte des Evangeliums von der Liebe Christi nötig, damit durch Mitmenschlichkeit, praktische und sozial-philanthropische Zusammenarbeit im Blick auf die vielfältigen Leiden der Menschen die gnädige und großzügige Liebe der Heiligen Dreifaltigkeit geteilt werde. $^{8}$

In demselben Nachrichtenportal www.basilica.ro berichtet Aurelian Iftimiu in einer kurzen Notiz vom 1. Juni 2017 über die Verleihung der Ehrendoktorwürde an den Ökumenischen Patriarchen Bartholomäus durch die Fakultät für Protestantische Theologie der Universität Tübingen. Dieses am 30. Mai 2017 stattgefundene Ereignis hat, über die Würdigung eines hohen orthodoxen Würdenträgers - der sich für Frieden, Freiheit, soziale Gerechtigkeit und darüber hinaus für den Schutz der Umwelt in besonderer Weise verdient gemacht hat - hinaus, eine enorme Symbolkraft. Es ist sicherlich kein Zufall, dass im Jahr des 500. Reformationsjubiläums jene protestantische Fakultät den jetzigen Ökumenischen Patriarchen ehrt, deren Theologen Martin Crusius, Jakob Andreae und Lukas Osiander bereits im 16.

8 „Într-o Europă tot mai secularizată, tensionată și fragmentată spiritual este necesară o mărturisire comună a valorilor eterne ale Evangheliei iubirii lui Hristos pentru umanitate și o conlucrare practică, social-filantropică, în fața suferințelor multiple pentru a împărtăși oamenilor iubirea milostivă sau darnică a Preasfintei Treimi.“ Siehe: https://basilica.ro/diferiti-in-credinta-dar-pasnici-in-convietuire-ortodocsi-si-luterani-in-transilvania/, abgerufen am 21.09.2019. 
Das 500. Reformationsjubiläum in rumänisch-orthodoxer Wahrnehmung Jahrhundert (genauer zwischen den Jahren 1573 - 1581) einen Briefwechsel mit dem damaligen Ökumenischen Patriarchen Jeremias II. geführt haben. ${ }^{9}$

Es war eine der wichtigen ökumenischen Gesten des Reformationsjubiläumsjahres, welches die Evangelische Kirche ganz bewusst nicht in Abgrenzung von den andern Glaubensgeschwistern, sondern zusammen mit ihnen feiern wollte; eine Geste, die die Wertschätzung zeigt, die man sich gegenseitig entgegenbringt. Dass ein rumänisch-orthodoxes Nachrichtenmedium dieses in Deutschland stattgefundene Ereignis seinen Lesern präsentiert, hat Signalwirkung: es zeigt an, dass der ökumenische Dialog weitergeht und dass diese Fortführung von beiden Seiten gewünscht ist.

Wie es zu erwarten war, bringt die Fachzeitschrift der orthodoxen Fakultät aus Hermannstadt „Revista Teologică “10 ${ }^{10}$ gleich mehrere Artikel, die mit der Reformation und dem Reformationsjubiläum in Zusammenhang stehen.

Die beiden Professoren Aurel Pavel und Daniel Buda berichten in der Nr. 1/2017 dieser theologischen Fachzeitschrift über das ökumenische Treffen vom 31. Oktober 2016 in Lund ${ }^{11}$, wo Papst Franziskus mit dem Präsidenten des Lutherischen Weltbundes (LWB) Munib Younan und der schwedischen Erzbischöfin Ante Jackelen das Reformationsjubiläum eröffneten. Neben einigen Beobachtungen und Einschätzungen über dieses Treffen wird auch die vollständige Predigt von Papst Franziskus in rumänischer Übersetzung abgedruckt.

Die beiden Autoren referieren darüber, wie dieses Treffen bewertet werden soll. Einerseits zählt dieser Papstbesuch zu den Überraschungsgesten, mit denen Franziskus immer wieder aufwartet. Andererseits wird beobachtet, dass Schweden ein Land ist, in dem die Katholiken eine kleine Minderheit darstellen. Offenbar war eine solche Feier etwa in Deutschland, dem Ursprungsland der Reformation, wo aber auch die Katholische Kirche sehr stark ist, (noch) nicht möglich. Der Ort der Begegnung war trotzdem nicht zufällig ausgewählt worden: in Lund/Schweden wurde im Jahre 1947 der Lutherische Weltbund gegründet. Nicht unbeachtet bleibt die Geste der geschwisterlichen Umarmung zwischen Papst Franziskus und der Erzbischöfin aus Stockholm Antje Jackelen.

\footnotetext{
9 Evangelische Kirche in Deutschland (Hrg.): Wort und Mysterium. Der Briefwechsel über Glauben und Kirche 1573 bis 1581 zwischen den Tübinger Theologen und dem Patriarchen von Konstantinopel, Witten, Luther Verlag 1958.

${ }_{10}$ https://www.revistateologica.ro, abgerufen am 21.09.2019.

11 https://www.revistateologica.ro/wp-content/uploads/2018/03/Aurel-Pavel-Daniel-Buda-Comemorarea-ecumenic\%C4\%83-a-Reformei-Lund-Suedia-31-octombrie-2016.pdf, abgerufen am 21.09.2019.
} 
Weiterhin wird die Rede von Papst Franziskus einer Analyse unterzogen. Der Papst sprach in Anlehnung an das Gleichnis vom Weinstock und den Reben (Joh. 15) über die christliche Einheit. Der Garant dieser Einheit ist Jesus Christus, der selber der Weinstock ist, während wir Christen die Reben sind. Gott der Vater ist der Weingärtner und die christliche Einheit wächst unter seiner Vorsehung. So würdigte der Papst den lutherisch-katholischen theologischen Dialog, den die Katholische Kirche mit dem Lutherischen Weltbund seit 50 Jahre führt. Was in der Geschichte falsch gelaufen ist, darüber steht es nur Gott zu, ein Urteil zu fällen. Offenbar waren es aber die Machthaber dieser Welt (politische wie religiöse), die für die Trennung verantwortlich sind, und nicht das einfache Volk, in dem der Wille nach Einheit in natürlicher Form vorhanden ist. Weiterhin wichtig ist die positive Bewertung der Reformation Luthers durch Franziskus, jedenfalls die positivste Bewertung der Reformation, die je von einem Papst vorgenommen wurde. Die Wichtigkeit der Heiligen Schrift wird hervorgehoben, wie auch das Prinzip der „sola gratia“. Die Rechtfertigung - so wie der Papst Luther interpretiert - drückt die Essenz der menschlichen Existenz vor Gott aus. Jesus ist der, der als Mittler vor Gott sich für uns einsetzt.

Die Beobachtungen von Pavel und Buda schließen mit dem Hinweis, dass trotz dieser durchaus positiven Darstellung der Reformation durch Papst Franziskus, diese nach wie vor unter der Verwerfung durch Papst Leo X. durch die Bulle Exsurge Domine aus dem Jahr 1520 steht, und die Diskussion über eine Aufhebung der Anathema noch gar nicht begonnen hat.

Einen umfassenden Artikel publiziert ebenfalls in der Hermannstädter „Revista Teologică“ (Ausgabe Nr. 3/2017) Mihai Iordache, und zwar unter dem Titel: „Cauzele Reformei Protestante și noțiunea de libertate la marii reformatori: Martin Luther, Jean Calvin și Ulrich Zwingli“"12 („Die Ursachen der Reformation und der Freiheitsbegriff bei den drei großen Reformatoren Luther, Calvin und Zwingli“"). Der Autor möchte

[...] in objektiver Weise die realen Ursachen des Ausbruches der protestantischen Reform im Westen erforschen und der Bedeutung des Freiheitsbegriffes im Denken der drei großen Reformatoren Martin Luther, Jean Calvin und Ulrich Zwingli nachgehen. ${ }^{13}$

\footnotetext{
12 https:/www.revistateologica.ro/wp-content/uploads/2018/03/Mihai-Iordache-Cauzele-Reformei-Protestante-\%C8\%99i-no\%C8\%9Biunea-de-libertate.pdf, abgerufen am 21.09.2019.

13 Ibidem, S. 36: „Intenția acestui studiu este de a cerceta cu obiectivitate cauzele reale ale izbucnirii Reformei Protestante în Apus și de a descoperi importanța noțiunii de libertate pentru gândirea marilor reformatori, Martin Luther, Jean Calvin și Ulrich Zwingli.“
} 
Das 500. Reformationsjubiläum in rumänisch-orthodoxer Wahrnehmung

Bevor er die drei Reformatoren einzeln betrachtet, umreißt er in zwei Abschnitten den kulturell-historischen Kontext der westlichen Welt vor der Reformation, die Ursachen der Reformation und ihre Folgen. Iordache stellt gleich am Anfang eine steile These auf:

[...] dass eine Transitivitätsrelation in einer nihilistischen Übung der westlichen Kultur abgeleitet werden kann, die sich von ihrer mittelalterlichen Konnotationen durch die Renaissance loslöst, um nachher der Reformation Raum zu schaffen[...] Die Reformation ihrerseits löst sich von sich selber durch die Gegenreformation, um nachher im protestantischen Kapitalismus aufzugehen, der seinerseits von der Romantik des 19. Jahrhunderts überwunden wurde [...] Das Ganze endet in dem apotheotischen Diskurs Nieztsches [...] formulierten Vorstellung, der Mensch sei das Maß aller Dinge. ${ }^{14}$

Iordache geht davon aus, dass aus orthodoxer Sicht das Mittelalter keineswegs als dunkle Periode gewertet werden muss, die Renaissance und Reformation als nötig erscheinen ließen. Das Mittelalter war in der Lage, die Barbaren zu christianisieren, und hat es damit geschafft, das Abendland vor den destruktiven Kräften der Wandervölker zu bewahren. Durch die Satisfaktionslehre Anselm von Canterburys wurde dem Menschen des Mittelalters eine eher kontemplative als rationale Haltung im Blick auf das ewige Leben anerzogen. Komplikationen treten in dem Moment auf, als das Christentum ein Instrument der Macht wird.

Die Ursachen der Reformation gehen über die Debatten zu Glaubensthemen weit hinaus. Iordache beschreibt kurz die politischen Zusammenhänge im damaligen Westeuropa, nennt aber auch soziale Missstände (z. B. die hohe Sterblichkeit aufgrund von Epidemien) und ökonomische Gründe. Die innerkirchlichen Streitigkeiten (Papst und Gegenpapst, sowie die Entstehung der Anglikanischen Kirche) taten das Ihre dazu.

Sodann wendet sich Iordache den einzelnen Reformatoren zu. In einem ersten Abschnitt beschreibt er den jeweiligen historischen Ablauf der Dinge, während er dann in einem zweiten Abschnitt auf den Freiheitsbegriff der drei Reformatoren eingeht. Die historischen Details sind dem deutschen

14 Ibidem, S. 37: „Într-un exercițiu de aritmetică culturală contemporană, vom putea să deducem o relație de tranzitivitate, într-un exercițiu nihilist al culturii occidentale, care se dezice de conotațiile sale medievale prin Renaștere, pentru ca mai apoi, să dea loc Reformei, care reprezintă o reluare a nihilismului, chiar dacă vechiul ascetism medieval este substituit, în societatea puritană, de un ascetism intramundan'. Reforma se dezice și ea prin Contrareformă, pentru ca mai apoi aceasta din urmă să își găsească disoluția prin Capitalismul protestant, învins în sec. al XVIII-lea de Romantism [...] sfârșind apoteotic în discursul nietzschean [...] pentru a crea omul, ca măsură a tuturor lucrurilor." 
Leserpublikum grundsätzlich hinreichend bekannt. Interessant ist die Analyse des Freiheitsbegriffes bei den drei Reformatoren.

Die Beurteilung des Wittenberger Reformators sieht wie folgt aus: In der Gleichung der drei Soli (sola scriptura, sola gratia, solus Christus), bleibt der Mensch alleine vor seiner Unfähigkeit stehen; er ist also nicht in der Lage, sich zu befreien, und muss immer wieder zu den Quellen des Heils zurückkehren; eine Idee - so Iordache - die später auch bei Karl Barth auftaucht. So ist der Mensch auf Christus geworfen, der alles, was nötig war, getan hat, so dass der Mensch nichts mehr tun muss. An dieser Stelle wird der Unterschied (nicht nur zur katholischen, sondern auch) zur orthodoxen Theologie deutlich, welche die Frage stellt: Was soll der Mensch tun? Es ist spannend, wie der orthodoxe Theologe Iordache den Unterschied in der Beurteilung des Freiheitsbegriffes - im Blick auf das Verhältnis des Menschen zu Gott - zwischen Luther und der von der Patristik kommenden Orthodoxie festhält (sowohl an dieser Stelle, als auch am Ende des Beitrags): für Luther ist das Verhältnis des Menschen zu Gott mit einer „Herr-Knecht-Beziehung" vergleichbar und dieses Denken sieht Iordache bei vielen anderen protestantischen Theologen im Laufe der Zeit bis heute. Luther vergisst - so der Vorwurf - die Schönheit des „Vater-Kind-Verhältnisses“, wie es von den heiligen Vätern und der orthodoxen Spiritualität verstanden wurde. Inwiefern dieser Vorwurf haltbar ist, möchte ich an dieser Stelle hinterfragen: in den orthodoxen Gebeten habe ich viel öfters die Bezeichnung „Herr“ (für Gott) und „Knecht“ (für den Menschen) vorgefunden denn Vater und Kind. Andererseits mag Luther wohl mit den Konzepten „Herr“ und „Knecht“ gearbeitet haben, was von seiner Biografie her verständlich ist; in der heutigen Zeit sind im Protestantismus jedoch eher die Begriffe „Vater“ (für Gott) und „Kind“ (für den Menschen) gebräuchlich.

Bei dem Genfer Theologen Calvin - so Iordache - ist die Freiheit exklusiv eine Frage des Verstehens des Gesetzes und der Kraft Gottes im Leben des Menschen. Die Freiheit hat vor allem eine palliative Funktion (also die Beschwerden lindernd, aber nicht die Ursachen derselben bekämpfend), die das beladene Gewissen vor dem Zwang der Rechtfertigung schützt und die Einsicht nähert, dass das menschliche Tun immer ungenügend ist. Nur der freie Mensch, der sich dem Worte Gottes unterordnet und durch Jesus Christus in Ewigkeit gerechtfertigt und erwählt ist, lebt wahrhaftig und verantwortlich vor Gott. Dieser Freiheitsbegriff unterscheidet sich von dem orthodoxen noch viel grundlegender als jener Martin Luthers; die „doppelte Prädestination" leuchtet durch diese Argumentation durch.

Zuletzt kommt bei Iordache auch der Züricher Reformator ins Blickfeld. Bei Zwingli hat die Freiheit eine ganz besondere Bedeutung: Sie ist dem 


\section{Das 500. Reformationsjubiläum in rumänisch-orthodoxer Wahrnehmung}

Menschen von Gott durch Jesus Christus gegeben, wodurch der Mensch von der Knechtschaft der Sünde befreit wurde. Es besteht eine direkte Verbindung zwischen dieser Befreiung und seinem ewigen Erwähltsein durch Gott und seiner Rechtfertigung. Gott ist die Quelle der Freiheit, während Christus ihr einziger Vermittler und ihr Durchführer ist. Christliche Freiheit heißt totales Vertrauen in Gott zu haben, der den Menschen ihre Sünden vergeben hat. Die Freiheit des Menschen steht in unmittelbarer Verbindung zur Gewissensfreiheit und wird allein von der Heiligen Schrift begründet.

Am Schluss seiner Ausführungen hält Iordache nochmals zusammenfassend fest, dass bei allen drei Theologen der Freiheitsbegriff etwas mit dem Verständnis und der Annahme des Gebotes und der Kraft Gottes zu tun hat, während in der Orthodoxie das „Vater-Kind-Verhältnis“ im Vordergrund steht. Auch wenn der Autor dieses Artikels sich als Ziel die „objektiven Erforschung" gesteckt hat, so merken wir immer wieder, dass er nicht frei von Klischees und Vorurteilen ist, wenn er schließt:

Eine Sache ist klar: die drei Reformatoren, Martin Luther, Jean Calvin und Ulrich Zwingli haben die tiefgreifende und geheimnisvolle Lehre - voll theologischer und spiritueller Bedeutungen - der Kirche des Ostens nicht gekannt [...] Ansonsten hätte die Reformation wahrscheinlich nicht stattgefunden. ${ }^{15}$

Dass sich ein orthodoxer Theologe im Jahr 2017 mit den drei großen Reformatoren und ihrem Verständnis des Freiheitsbegriffs auseinandersetzt, ist eine begrüßenswerte Initiative. Allerdings sind es z. T. sehr verkürzte und einseitige Darstellungen, die einer so breit gefächerten Thematik dann doch nicht gerecht werden; zumindest nicht dem selbstgesteckten Ziel des Autors, „objektiv“ zu urteilen und die Dinge „real“ darzustellen.

In derselben Ausgabe Nr. 3/2017 findet man die rumänische Übersetzung der acht sogenannten „Invokavitpredigten“ Martin Luthers aus dem Jahr 1522 mit einer Einleitung, verfasst von der Doktorandin der Hermannstädter orthodoxen Fakultät Maria Curtean. ${ }^{16}$ Diese Predigten sind dem deutschen Fachpublikum wohl bekannt. Für die rumänischsprachigen Leserinnen und Leser ist diese Übersetzungsarbeit jedoch ein wichtiger Beitrag zum besseren Kennenlernen der Theologie Martin Luthers.

15 Ibidem, „Un lucru este cert: cei trei reformatori, Martin Luther, Jean Calvin și Ulrich Zwingli, nu au cunoscut învățătura profundă și tainică, plină de semnificații teologice și spirituale a Bisericii Ortodoxe a Răsăritului [...] Probabil că, în caz contrar, Reforma nu ar mai fi avut loc $[\ldots]^{\text {“, }, ~ S . ~} 59$.

16 https://www.revistateologica.ro/wp-content/uploads/2018/03/Maria-Curtean-Optpredici-ale-Dr.-Martin-Luther-rostite-de-c\%C $4 \% 83$ tre-el-\%C3\%AEn-Wittenberg\%C3\%AEn-vremea-Postului-1522.pdf, abgerufen am 21.09.2019. 
Interessant ist aber nicht nur die Übersetzungsarbeit, die an und für sich einen großen Wert darstellt, sondern auch die geschichtliche Einleitung und eine kurze Zusammenfassung der Predigten, welche Curtean dazu liefert. Die orthodoxe Theologin vermittelt einen guten Überblick darüber, wie infolge des ersten „evangelischen“ Gottesdienstes zu Weihnachten 1521 die Ereignisse in Wittenberg sich zu überschlagen begannen und schließlich am Anfang des Jahres 1522 ein großes Chaos in der Stadt entstand. Diese Umstände bewogen Martin Luther dazu, am 6. März 1522 aus seinem sicheren Versteck von der Wartburg nach Wittenberg zurückzukommen und durch die vorhin genannten "Invokavitpredigten“ (sie wurden vom Sonntag „Invokavit“ beginnend bis zum darauffolgenden Sonntag „Reminiszere“ von Luther täglich gehalten) dahingehend zu wirken, dass wieder Ordnung einkehrte. Luther reiste bekanntlich am 2. April 1521 nach Worms und war - nachdem er sich dort geweigert hatte, seine Lehren zu widerrufen und sich damit in Todesgefahr gebracht hatte - anschließend auf die Wartburg gebracht worden. Nach seinem Weggang aus Wittenberg hatte dort sein ehemaliger theologischer Lehrer Andreas Bodenstein Karlstadt das Heft in die Hand genommen. Bereits im Frühjahr 1521 waren einige Pfarrer in den Ehestand getreten. Eine ganze Reihe von Messen war abgeschafft worden. Das Abendmahl wurde in beiderlei Gestalt gereicht. Ein gewaltiger Bildersturm setzte ein und Seitenaltäre wurden gewaltsam entfernt. Dazu kamen dann auch noch die sogenannten „Zwickauer Propheten“: Wiedertäufer aus dem Kreise Thomas Müntzers, die mit ihren apokalyptischen Vorstellungen, die auch den Gebrauch des Schwertes legitimierten, noch mehr Verwirrung stifteten. Jene, denen das alles zu schnell und zu brutal ging - vor allem einige Mönche aus den drei Klöstern Wittenbergs - wurden einfach überrannt oder ausgelacht.

All diese Ereignisse werden von Curtean kurz und kompakt beschrieben. 500 Jahre nach Luthers Thesenveröffentlichung wird mit diesem Beitrag ein wichtiges Kapitel Reformationsgeschichte in rumänischer Sprache zugänglich.

Der Bukarester Theologe Cosmin Pricop meldet sich gleich zweimal in ausländischen deutschsprachigen Publikationen zu Wort. In „Una Sancta - Zeitschrift für Ökumenische Begegnung“ publiziert er den Aufsatz „Reformation und Reformationsjubiläum aus orthodoxer Perspektive“, dem wir uns des Weiteren zuwenden. ${ }^{17}$

In diesem Aufsatz geht es um eine allgemeine Beurteilung der Reformation und des Reformationsjubiläums durch einen ökumenisch aufge-

17 Cosim Pricop, „Reformation und Reformationsjubiläum aus orthodoxer Perspektive“, in: Una Sancta - Zeitschrift für Ökumenische Begegnung 72 (3/2017), S. 184-194. 
Das 500. Reformationsjubiläum in rumänisch-orthodoxer Wahrnehmung

schlossenen orthodoxen Theologen. Bereits in der Einleitung lässt der Autor uns wissen, dass die orthodoxen Stellungnahmen zu Luther, Reformation und Protestantismus unterschiedlich, ja sogar gegensätzlich sind. Pricop erklärt das einerseits mit der Unfähigkeit der Orthodoxie, sich mit Luther und dem protestantischen Gedankengut auseinanderzusetzen; andererseits aber auch damit, dass eine Vielfalt von Deutungen der Theologie Luthers möglich ist. Während die einen den Wert der Theologie Luthers hervorheben, sehen andere in dem, was er hinterlassen hat, häretisches Gedankengut. Diese zweite Art der Beurteilung - wie wir sie übrigens weiter oben bei Iordache vorfanden kann nur zu dem Schluss führen: „Die Orthodoxie soll glücklich sein, dass es in ihrem Rahmen zu keinem Luther und zu keiner Reform gekommen sei." 18 Luther, Reformation und Protestantismus werden in orthodoxer Perspektive gerne dem „Westen“ zugeordnet, wobei - so Pricop - es ein Teil des gemeinsamen christlichen Erbes ist, mit dem man ins Gespräch kommen muss.

Nach dieser Einleitung erwähnt Pricop die wichtigen akademischen Veranstaltungen, die in diesem Zusammenhang stattgefunden haben:

- die (weiter oben bereits erwähnte) Verleihung der Ehrendoktorwürde an den Ökumenischen Patriarchen Bartholomäus durch die Evangelisch-Theologische Fakultät Tübingen; ${ }^{19}$

- die Veranstaltung der Ludwig-Maximilians-Universität München zum Thema: „500 Jahre Reformation - Wo steht die Ökumene?“, die sich über drei Semester lang jeweils mit der katholischen, protestantischen und orthodoxen Perspektive auseinandersetzte;

- die internationale Tagung „1517 - 2017. 500 Years of Reformation“, welche von der Aristoteles Universität und dem Deutschen Konsulat in Thessaloniki ${ }^{20}$ organisiert worden war;

- das Symposium des Institutes für Ökumenische Forschung in Hermannstadt. ${ }^{21}$

Eine wichtige Verbindung zwischen dem Reformationsjubiläum aus dem Jahr 2017 und dem ein Jahr vorher stattgefundenen Panorthodoxen Konzil von Kreta stellt Pricop her, indem er auf eines der Konzilsdokumente verweist: „Die Beziehungen der Orthodoxen Kirche zur übrigen christli-

18 Ibidem, S. 185.

19 Ibidem, S. 186: Pricop interpretiert sie „[...] als ein symbolischer Akt der Aktualisierung und zugleich Fortsetzung ursprünglicher, im 16. Jahrhundert geknüpfter Kontakte zwischen den Tübinger Anhängern der Reformation - Theologen und Philosophen - und dem damaligen Patriarchen von Konstantinopel [...]“.

20 Ibidem, S. 186. Pricop nennt sie: „[...] die signifikanteste Unternehmung innerhalb der orthodoxen Welt auf akademischem Niveau hinsichtlich der Wahrnehmung der lutherischen Reformation [...]“.

${ }^{21}$ Review of Ecumenical Studies 9 (3/2017). 
chen Welt. “"22 Die Verantwortung der Orthodoxen Kirche für die Einheit der Christen wird in aller Deutlichkeit hervorgehoben. Pricop verschweigt nicht, dass es innerhalb der Orthodoxie skeptische Sichtweisen, aber auch ultra-konservative und anti-ökumenische Kreise gibt, denen die sowieso schon vorsichtig gehaltenen Formulierungen zu weit gehen. Trotzdem wird daran festgehalten, dass die bilateralen Dialoge fortgeführt werden sollen, wenn auch unter bestimmten Bedingungen; z. B., dass sie keine kirchliche Gemeinschaft voraussetzen, da die Gleichheit zwischen den Konfessionen als solche abgelehnt wird. Die orthodoxe Perspektive im Blick auf ihre Beziehung zur christlichen Welt wird unter Zuhilfenahme der Begriffe „schon“ und „trotzdem" dargestellt:

Der Begriff Schon bringt die innerhalb der Orthodoxie formulierte Überzeugung zum Ausdruck, derzufolge die Wiederherstellung der Einheit der Christen, die Teilnahme der orthodoxen Lokalkirchen an bilateralen und internationalen ökumenischen Dialogen und Initiativen usw. als notwendig und wünschenswert zu betrachten seien. Auf der anderen Seite lässt sich mittels des Begriffs Trotzdem die Einsicht der Orthodoxie entschlüsseln, dass die gewünschte Einheit der Christen nicht vorschnell und oberflächlich zu realisieren wäre, sondern auf der Basis deren Entsprechung mit dem Glauben der Einen, Heiligen, Katholischen und Apostolischen Kirche, wie er innerhalb der Orthodoxie zu finden sei. ${ }^{23}$

Sodann gibt er eine Übersicht über den internationalen theologischen lutherisch-orthodoxen Dialog, wobei er in einer übersichtlichen und anschaulichen Tabelle die abgearbeiteten Dialogthemen nennt, sowie die Übereinstimmungen und Unterschiede festhält. ${ }^{24}$

Dieser Beitrag schließt mit dem Fazit, dass vor dem Hintergrund einer langen Gesprächstradition mit dem Luthertum die Orthodoxe Kirche sich auch weiterhin in theologischen Dialogen engagieren wird und sich in Verantwortung ihren Gesprächspartnern gegenüber sieht. Dabei geht es nicht nur darum, die andere Seite mit ihren theologischen Eigenschaften kennenzulernen, sondern Impulse zur Selbstreflexion und Selbstkorrektur zu gewinnen. Das Ganze „[...] aus Respekt für die Vorgänger, die viel daran gearbeitet haben, und in der Überzeugung, dass die Reformation nicht nur den Protestanten gehört, sondern der ganzen Christenheit“ ${ }^{25}$

\footnotetext{
22 http://basilica.ro/sfantul-si-marele-sinod-relatiile-bisericii-ortodoxe-cu-ansamblul-lumii-crestine-document-oficial/, abgerufen am 04.10.2019.

23 C. Pricop, „Reformation und Reformationsjubiläum “, S. 189.

${ }^{24}$ Ibidem, S. 192-193.

25 Ibidem, S. 194.
} 


\section{Das 500. Reformationsjubiläum in rumänisch-orthodoxer Wahrnehmung}

Für den Sammelband „Sola Scriptura 1517 - 2017“ verfasste Cosmin Pricop den Beitrag „Sola scriptura? Eine rumänisch-orthodoxe Perspektive" ${ }^{26}$ Es ist sicher der theologisch fundierteste Beitrag eines orthodoxen Theologen zu einem genuin reformatorischen Thema im Zusammenhang des 500. Reformationsjubiläums, auf den ich gestoßen bin, wobei der Autor einerseits das Ganze mit einer beeindruckenden ökumenischen Offenheit angeht, andererseits aber in wissenschaftlicher Redlichkeit das Wesentliche anspricht. Er ist sich dessen bewusst, dass er sich mit seinem Nachdenken über „sola scriptura“ erst am Anfang eines Weges befindet, von dem zu hoffen ist, dass er auch von anderen orthodoxen Theologen beschritten wird.

Wir haben es hier - nach eigenem Bekunden von Pricop - nicht mit einer „traditionellen orthodoxen Position“ ${ }^{27}$ zu tun. Der Autor selber be-

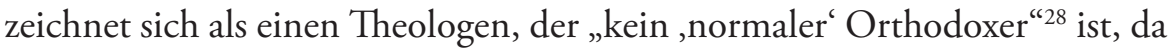
er in den eignen Reihen mitunter der „Verwässerung des einzigen richtigen Glaubens beschuldigt " ${ }^{29}$ wird; wohl aber ist er einer, der „den Reichtum der akademischen Bibelwissenschaft entdeckte "30 und von daher ist seine Herangehensweise zu verstehen. Pricop gehört zu den orthodoxen Theologen, die die Überzeugung teilen, dass es im ökumenischen Dialog kein Weiterkommen gibt, wenn man immer wieder die alten Klischees und Feindbilder bemüht. Und so beginnt sein Beitrag thesenartig:

Das theologische Konzept von sola scriptura ist meines Erachtens für alle Theologinnen und Theologen bedeutend, egal aus welchem theologischen Kontext sie stammen und zu welcher christlichen Konfession sie gehören. Die tatsächliche Komplexität des zu erörternden Themas lässt sich - zumindest für die orthodoxe Bibelperspektive - nicht wirklich erahnen. Im Laufe der Untersuchung aber wurde meine bisherige Überzeugung umso mehr bekräftigt, der zufolge Luther, Protestantismus und sola scriptura nicht nur ein Merkmal des Westens sind [...] sondern Bestandteile unseres christlichen Erbes sind, mit denen es sich auseinanderzusetzen gilt, um sie besser kennenzulernen und gegebenenfalls um die eigene Sichtweise zu ergänzen. ${ }^{31}$

Pricop möchte die gängigen Positionen und Stellungnahmen der „normalen“, also konservativen Orthodoxie hinsichtlich der Beurteilung des

26 Idem, „Sola scriptura? Eine rumänisch-orthodoxe Perspektive“, in: Stefan Alkier (Hrg.), Sola Scriptura 1517-2017, Tübingen, Mohr Siebeck 2019, S. 359-376.

27 Ibidem, S. 359.

28 Ibidem, S. 360.

29 Ibidem.

30 Ibidem.

31 Ibidem, S. 359. 
Protestantismus im Allgemeinen und des theologischen Konzeptes von „sola scriptura" im Speziellen zurücklassen, um sich dem Thema unbefangen nähern zu können. Im Gegensatz zu Iordache beansprucht er weder „objektiv“ zu urteilen, noch die Dinge „real“ darzustellen.

Im ersten Teil seines Beitrags wirft Pricop einen Blick auf die „Geschichte der Wahrnehmung und Auseinandersetzung mit dem Schriftprinzip sola scriptura auf Seiten der orthodoxen Theologie“32. Eingeleitet wird der historische Überblick mit dem Hinweis auf den bereits erwähnten Briefwechsel zwischen den Tübinger Theologen mit dem Ökumenischen Patriarchen aus dem 16. Jahrhundert. Es ist bekannt, dass dieser Briefwechsel im Jahr 1581 von Patriarch Jeremias II. abgebrochen wurde. Ein wesentlicher Grund für das Scheitern dieses Dialogs waren wohl die verschiedenen Haltungen zu Schrift und Tradition der Dialogpartner, wobei dieses Scheitern aber „[...] auf die mangelnde Methodologie, andererseits aber auf das begrenzte Wissen über das jeweilige theologische Profil des Gesprächspartners sowie auf die stark differierenden Erwartungen zurück[zu]führen "33 sind. Der Stand des Dialogs im 16. Jahrhundert ist dieser: „Der Patriarch hebt stetig die Bedeutung der kirchlichen Tradition hervor und die Tübinger beharren auf der Priorität der Schrift und auf deren Rolle für die Bewertung der Tradition. " ${ }^{34}$ Die Feststellung von Pricop ist aber jene, dass die beiden Seiten gar nicht so weit auseinander liegen:

Irgendwie bekommt man in diesem Zusammenhang den Eindruck, dass man es hier mit verschiedenen, aber nicht gegensätzlichen Betonungen derselben Offenbarungsgeschichte Jesu Christi zu tun hat: Die einen sehen sich genötigt, zu Beginn und Ursprung dieser Geschichte zu kommen und aus dem dort gewonnenen Standpunkt zu argumentieren, während die anderen eher die Fortsetzung und Vergegenwärtigung dieser Offenbarung zu erläutern beabsichtigen. ${ }^{35}$

Es ist wichtig festzuhalten, dass aus der Sicht von Pricop diese Art des Umgangs mit Theologie auch für die heutige Zeit ersprießlich ist. Zugleich aber ist er sich dessen bewusst:

[...] dass auch heutzutage, wenn ein orthodoxer Bischof eine offizielle kirchliche Antwort auf ein strittiges theologisches Thema schriebe, sich diese nicht bedeutsam von der Jeremias II. unterscheiden würde $[\ldots] .{ }^{36}$

32 Ibidem, S. 361.

33 Ibidem, S. 362.

4 Ibidem, S. 365.

35 Ibidem.

36 Ibidem. 
Das 500. Reformationsjubiläum in rumänisch-orthodoxer Wahrnehmung

In der Zeitspanne zwischen dem Briefwechsel der Tübinger Theologen mit dem Ökumenischen Patriarchen aus dem 16. Jahrhundert und dem Beginn der ökumenischen Kontaktaufnahmen des 20. Jahrhunderts sieht Pricop keine erwähnenswerten Momente, in denen Orthodoxe und Protestanten sich theologisch nähergekommen wären. In einer kurzen Beschreibung der ökumenischen orthodox-lutherischen Dialoge des 20. Jahrhunderts vermerkt Pricop, dass das Thema "Schrift und Tradition“ gleich mehrere Male thematisiert wurde, wobei „[...] das Schriftprinzip sola scriptura in einer mehr integrativen Weise durch die Lutheraner verstanden wird [...]",37 was der Orthodoxie entgegenkommt. Sodann erwähnt er drei Forschungsarbeiten orthodoxer Autoren zu dem Thema Schrift und Tradition: jene von Viorel Mehedinţiu, ${ }^{38}$ „Offenbarung und Überlieferung. Neue Möglichkeiten eines Dialogs zwischen der orthodoxen und evangelisch-lutherischen Kirche“, Mihail Staikos, ${ }^{39}$ „Sola Scriptura sine traditione? Aktuelle Perspektiven über Schrift und Tradition im Ökumenischen Dialog zwischen der Orthodoxie und dem Lutherischen Weltbund“ und John Whiteford, ${ }^{40}$ "Sola Scriptura: An Orthodox Analysis ofthe Cornerstone of Reformation Theology".

In dem Zwischenergebnis hält Pricop als Schlussfolgerungen fest, dass die orthodoxe Theologie bereits seit dem 16. Jahrhundert und bis heute die protestantische Lehre und die Identität der Protestanten in engem Zusammenhang mit dem Begriff „sola scriptura“ sieht:

Unter sola scriptura verstanden und verstehen die Orthodoxen denjenigen protestantischen Lehrsatz, dem zufolge die Bibel die höchste Autorität in Glaubensfragen hätte und dadurch die Tradition der Kirche als zweitrangig unterbewertet wäre. ${ }^{41}$

Auch heute noch werden Protestantismus und protestantisches Bibelverständnis in der Orthodoxie aus dem Blickwinkel des Briefwechsels der Tübinger Theologen mit dem Ökumenischen Patriarchen gesehen und verstanden. Der Befund der Wahrnehmungsgeschichte von Pricop ist jener,

[...] dass die orthodoxe Theologie sich mit dem Schriftverständnis der Reformation niemals ernsthaft auseinandergesetzt hat [...] Im Rahmen ökumenischer Gespräche beschäftigt sich kein orthodoxer Theologe mit der gründlichen Analyse des sola scriptura-Prinzips. ${ }^{42}$

\footnotetext{
37 Ibidem, S. 366.

38 Ibidem, S. 367.

39 Ibidem.

40 Ibidem, S. 368.

${ }^{41}$ Ibidem, S. 369.

42 Ibidem.
} 
Pricop identifiziert auch die Ursache für diese Art von Umgang, die wohl darin liegt,

[...] dass die Teilnehmer bei der Annäherung an die Themen

Schrift und Tradition lediglich die jeweilige Positionen der eigenen

Kirche bzw. Konfession darstellen, ohne dabei Interesse für die

Perspektiven der Gesprächspartner zu zeigen. ${ }^{43}$

Weil in der Orthodoxie bis auf den heutigen Tag ein konstruktiver Umgang aufgrund wissenschaftlicher Untersuchungen mit dem Begriff „sola scriptura" fehlt, wird er insgesamt als wesensfremd angesehen.

Im zweiten Teil seines Beitrags versucht Pricop anhand der Analyse einer Schrift Luthers ${ }^{44}$ „Assertio Omnium Articulorum M. Lutheri, per Bullam Leonis X novissimam damnatorum“ das Prinzip „sola scriptura" in einen Dialog mit seiner orthodoxen Position zu bringen. Dabei identifiziert er drei „Annäherungspunkte“ 45 und drei „offene Aspekte“.

Die „Annäherungspunkte“ sind die folgenden:

- Als Bibelwissenschaftler weiß Pricop, wovon er spricht, wenn er die „protestantische Expertise“46 ins Feld führt, an der man in der heutigen Zeit nicht mehr vorbeikommt, wenn man biblische Theologie nach wissenschaftlichen Kriterien betreibt. Zwar ist er sich nicht sicher, ob diese Fokussierung auf die Heilige Schrift dem sola scriptura geschuldet ist. Ich würde die Frage umkehren: Wem denn sonst?

- Ein weiterer Annäherungspunkt ist die Kritik Luthers an der Institution Kirche und von daher kommend die Hochhaltung der Laien (Priestertum aller Getauften), die ihr Pendant auf der orthodoxen Seite in dem „Phänomen der ,geistlichen Väter ${ }^{6}$ und ,geistlichen Mütter “ ${ }^{\text {‘7 } 7}$ hat. Das hat nur indirekt etwas mit dem Prinzip „sola scriptura“ zu tun, in dem Sinne, dass die kirchliche Tradition für diese Menschen nicht mehr den Stellenwert hat wie ehedem: Sowohl die einen als auch die andern haben einen unmittelbaren Zugang zu Gott, ohne besonderer kirchlicher Traditionen zu bedürfen.

- Einen dritten Anknüpfungspunkt sieht Pricop in der Ermutigung für die Orthodoxen, das biblische Wort direkt wahrzunehmen,

\footnotetext{
${ }^{43}$ Ibidem.

44 Ibidem, S. 370.

45 Ibidem.

46 Ibidem, S. 371.

47 Ibidem.
} 
Das 500. Reformationsjubiläum in rumänisch-orthodoxer Wahrnehmung

also die Bibel selber zu lesen. Damit setzt Pricop sich von dem Mainstream in der heutigen Orthodoxie ab, in der - genau das kritisierte auch Luther seinerzeit - weniger die Bibel selbst, sondern die patristischen Kommentare dazu gelesen werden: „Man traut sich nicht mehr, seine eigene Erfahrung mit dem Bibeltext zu gewinnen und zu genießen und denkt, dass der Ausdruck, meiner Meinung nach', geäußert im Kontext der Erschließung biblischer Texte, ein deutlicher Hinweis auf die protestantische Herkunft der jeweiligen Exegeten sei. “48 Es geht nichts über die Erfahrung mit dem Text, die jedoch natürlich nicht verabsolutiert werden kann, sondern jeweils konkret im Leben aktualisiert werden muss.

Es folgen die ,offenen Aspekte', die ich persönlich sehr anregend und weiterführend für den interkonfessionellen Dialog zwischen Orthodoxie und Luthertum finde:

- Es ist nicht so sehr der Begriff „scriptura“, sondern das „sola“, welches irritiert bzw. eine "'orthodoxe' Sensibilität" ${ }^{49}$ auf den Plan ruft. Die Bibel wird in der Orthodoxie nicht unterbewertet. Die von ihr beschriebenen Gotteserfahrungen gehen aber über ihren Rahmen hinaus; sie setzten sich durch die Kirchengeschichte fort bis in die heutige Zeit. „Durch jede Bibellektüre wird das Erreichen des Zugangs zu den ursprünglichen Erfahrungen, Begegnungen und Ereignissen beabsichtigt. [...] Demzufolge fungiert die Bibel nicht als ultimative Instanz oder Referenz. Sie hilft dem Menschen, ursprüngliche gott-menschliche Erfahrungen zur Kenntnis zu nehmen, ist allerdings nicht als Ziel in ipse zu begreifen. "50 Bemerkenswerter Weise ist die Orthodoxie ihrerseits auch von einer „sola“-Perspektive berührt: die „sola traditio“. Die orthodoxe Theologie nimmt immer wieder auf die Kirchenväter Bezug, ja mehr noch: „Die Heiligen Kirchenväter fungieren als ultimative Begründung [...] was meiner Meinung nach nicht im Sinne der Kirchenväter sein kann." ${ }^{\text {"1 }}$

- Pricop erinnert daran, dass die orthodoxe Theologie ursprünglich keine Unterscheidung und auch keine Trennung zwischen Schrift und Tradition vorgenommen hat. Wenn das die orthodoxe Kirche heute tut, ist dies eher dem abendländischen Einfluss geschuldet.

48 Ibidem, S. 372.

49 Ibidem, S. 373.

50 Ibidem, S. 374.

51 Ibidem, S. 374-375. 
"Sowohl in der Bibel als auch in der kirchlichen Tradition geht es eigentlich und hauptsächlich um dieselbe schon erwähnte Kenntnisnahme der Begegnungen, Erfahrungen und Ereignisse Gottes mit den Menschen. "52 Pricop stellt hier einen feinen, aber wesentlichen Unterschied zwischen dem protestantischen und dem orthodoxen Blick auf die Heilige Schrift heraus. In protestantischer Sicht ordnen sich die Kirchenväter der Heiligen Schrift unter, weil sie diese lesen und interpretieren. In orthodoxer Sicht geht es aber bei der patristischen Lektürepraxis „[...] nicht einfach um eine Aktualisierung der Bibel, sondern eine Aktualisierung der in der Bibel beschriebenen Erfahrungen, Begegnungen und Ereignisse [...] "53 Bibellesen in patristischer Tradition bedeutet eben nicht nur eine Reise in die Vergangenheit, sondern eine Rückkehr in die Gegenwart, also Aktualisierung und Rekontextualisierung.

- Der Beitrag schließt mit dem Hinweis auf die Heilige und Göttliche Liturgie, wo nach orthodoxem Verständnis die Aktualisierung und Rekontextualisierung der biblischen Ereignisse, Begegnungen oder Erfahrungen stattfindet. Die Liturgie hat einen anamnetischen Teil, der dem Weg in die Vergangenheit entspricht; die biblischen Ereignisse, Begegnungen oder Erfahrungen werden erinnert. Der andere epiklektische Teil dann entspricht der Rückkehr in die Gegenwart, in dem vergegenwärtigt wird. So wird die Heilige Schrift zusammen mit den Bildern und den Hymnen zu einem Medium, welches einen persönlichen Zugang zu Gott vermittelt.

III.

Auch wenn der Protestantismus allgemein und das 500. Reformationsjubiläum im Speziellen nicht Hauptbeschäftigungsgebiet der meisten orthodoxer Theologen sind, so bieten die vorgestellten Beiträge ein buntes Mosaik, welches von der Berichterstattung über wichtige Ereignisse aus dem Jahr 2017 über die (mehr oder weniger konstruktive) Auseinandersetzung mit theologischen Themen reicht.

Im Vergleich zu der Berichterstattung und der Auseinandersetzung mit dem 450. Jubiläum fällt auf, dass dieser „Strauß“ an Beiträgen bunter ist. An den beiden letzten Artikeln von Cosmin Pricop wird einer der wesentlichen Unterschiede zwischen der aktuellen Situation und jener von 1967 ersichtlich, nämlich dass rumänisch-orthodoxe Theologen heute ungehindert und

\footnotetext{
52 Ibidem, S. 375.

53 Ibidem.
} 
Das 500. Reformationsjubiläum in rumänisch-orthodoxer Wahrnehmung

unzensiert im Ausland publizieren können; so etwas wäre vor 1989 so nicht vorstellbar gewesen. Diese beiden Artikel - vor allem jener, in dem er sich mit dem Begriff „sola scriptura“ auseinandersetzt - sind wichtige Beiträge für das weitere ökumenische Gespräch zwischen Orthodoxen und Protestanten.

Im Vergleich zum Jahr 1967 - die wichtigen orthodoxen Publikationen im damaligen kommunistischen Rumänien (allesamt Printmedien) können an den Fingern abgezählt werden - gibt es im heutigen Umfeld der rumänischen Orthodoxie eine Fülle an Publikationen, die als Printmedien zugänglich oder online abrufbar sind. Dazu kommen der landesweit ausstrahlende Fernsehsender TRINITAS und mehrere lokale Radiosender, von orthodoxen Eparchien betrieben, die nicht Gegenstand meiner Untersuchung waren.

Etwas verwundert war ich darüber, dass ich in den drei wichtigen Bukarester Publikationen, die im Jahr 1967 das 450. Reformationsjubiläum mehr oder weniger ausführlich thematisiert haben, das 500. Reformationsjubiläum betreffend nicht fündig geworden bin. Genauer gesagt: im Amtsblatt „Biserica Ortodoxă Română” wurde das Ereignis gar nicht erwähnt, die Ausgaben des Jahres 2017 der Zeitschrift der Bukarester Orthodoxen Fakultät „Studii Teologice“ sind noch nicht erschienen, während die Webseite des Patriarchates „Ortodoxia“ sich im Aufbau befindet ${ }^{54}$. Ebenso sprechen die Zeitschriften zweier wichtiger Orthodoxer Theologischen Fakultäten (wo man es vielleicht hätte erwarten können) das Thema nicht an, nämlich die Fachzeitschrift „Teologie și Viață“ aus Iaşi, wie auch die Fachzeitschrift „Studia Theologia Orthodoxa“ der Klausenburger Fakultät. Ein plausibler Grund, das Reformationsjubiläum nicht an vorderster Stelle auf der Prioritätenliste zu haben, war sicherlich das Jahrhundertereignis der Orthodoxie, nämlich das Panorthodoxe Konzil, welches zu Pfingsten 2016 auf Kreta stattgefunden hatte und mit dessen theologischen Inhalten und seiner Aufarbeitung sich die orthodoxen Fachzeitschriften bzw. Theologen in der Zeit danach - vor allem im Jahr 2017 - auseinandersetzten.

Dass aber orthodoxe Print- und Onlinemedien über das Reformationsjubiläum überhaupt berichtet haben und orthodoxe Theologinnen und Theologen sich mit "protestantischen" Themen auseinandergesetzt haben, ist ein deutliches Zeichen dafür, dass der Protestantismus vom rumänisch-orthodoxen „Radarschirm“ nicht verschwunden ist. Das ist gut so und ein Zeichen dafür, dass der ökumenische Dialog weitergeht. In diesem Sinne wäre es genauso wünschenswert, dass protestantische Medien sich mit orthodoxen Themen auseinandersetzen.

54 Stand am 4. Oktober 2019. 